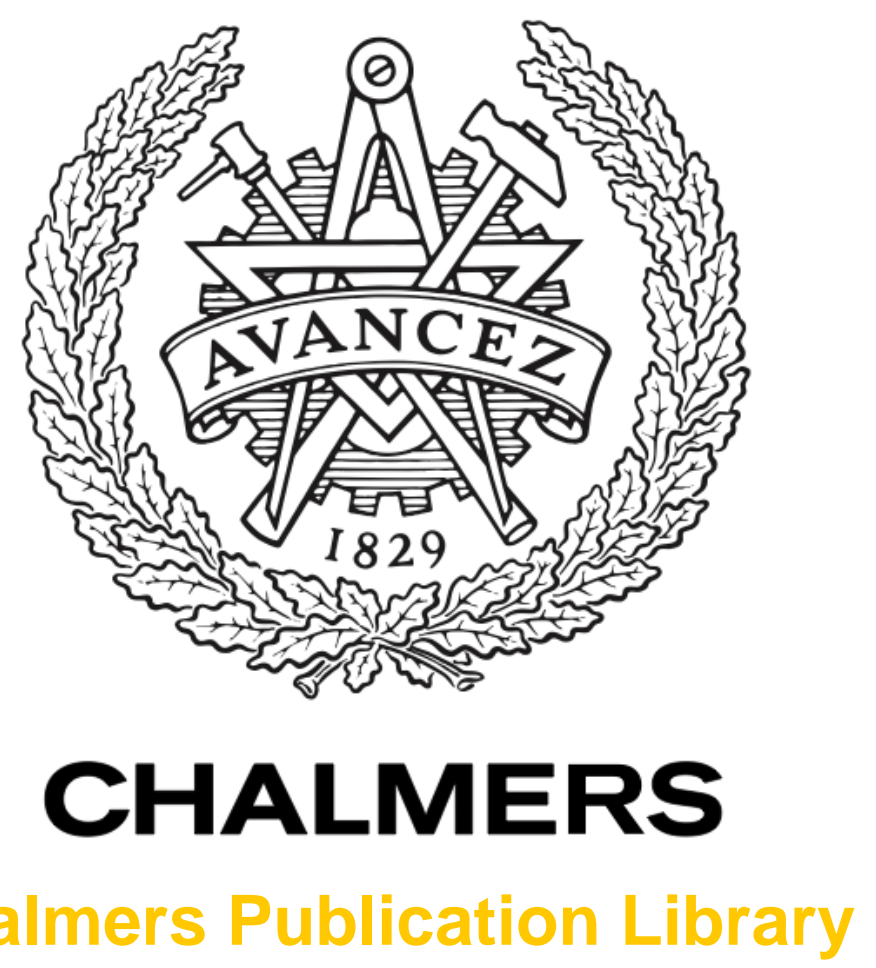

\title{
Bandlimited Power-Efficient Signaling for Intensity Modulation
}

This document has been downloaded from Chalmers Publication Library (CPL). It is the author's version of a work that was accepted for publication in:

ECOC, Sept. 2014, Cannes

Citation for the published paper:

Czegledi, C. ; Khanzadi, M. ; Agrell, E. (2014) "Bandlimited Power-Efficient Signaling for Intensity Modulation". ECOC, Sept. 2014, Cannes

Downloaded from: http://publications.lib.chalmers.se/publication/200779

Notice: Changes introduced as a result of publishing processes such as copy-editing and formatting may not be reflected in this document. For a definitive version of this work, please refer to the published source. Please note that access to the published version might require a subscription. 


\title{
Bandlimited Power-Efficient Signaling for Intensity Modulation
}

\author{
Cristian B. Czegledi ${ }^{(1)}$, M. Reza Khanzadi ${ }^{(1,2)}$, and Erik Agrell ${ }^{(1)}$ \\ (1) Department of Signals and Systems, czegledi@chalmers.se \\ (2) Department of Microtechnology and Nanoscience, \\ Chalmers University of Technology, Gothenburg, SE-41296, Sweden.
}

\begin{abstract}
A new, power-efficient signaling method for intersymbol interference-free transmission over the bandlimited intensity-modulation direct-detection channel is proposed. The method utilizes pulse-amplitude modulation with a sinusoidal bias function and is more power-efficient than previously known methods.
\end{abstract}

\section{Introduction}

Intensity-modulation direct-detection (IM/DD) systems are a potential solution for low-cost, low-complexity optical communication links. In such systems, the information is encoded on the intensity of the transmitted optical signal by varying the driving current of a verticalcavity surface-emitting laser, a laser diode, or a lightemitting diode at the transmitter. Direct detection is performed at the receiver by using a photodetector that generates an electrical current, proportional to the received optical power ${ }^{1}$. Applications of IM/DD systems include short-range optical links such as fiber-to-thehome, optical interconnects ${ }^{2}$, and diffuse indoor wireless optical links ${ }^{1}$. An IM/DD system implies two major constraints on the transmitted electrical signal; it must be nonnegative, and for safety and energy consumption purposes, both average and peak optical power have to be within certain limitations ${ }^{1}$.

Hranilovic investigated ${ }^{3}$ intersymbol interference (ISI)-free transmission over a strictly bandlimited IM/DD channel for the first time. Pulse-amplitude modulation (PAM) schemes were designed using bandlimited ISI-free nonnegative Nyquist pulses such as the squared sinc (S2) pulse, which requires a bandwidth equal to the symbol rate. It was also shown that nonnegative bandlimited ISI-free root-Nyquist pulses do not exist. As an extension, new nonnegative Nyquist pulses were introduced ${ }^{4}$, which provide a trade-off between the required optical power and the bandwidth. In $2012^{5}$, a new modulation scheme for bandlimited ISI-free IM/DD systems was proposed, where a directcurrent (DC) bias signal is added to the transmitted waveform in order to make it nonnegative. This approach improves the bandwidth efficiency, by reducing the required bandwidth below the symbol rate, and it also allows the usage of root-Nyquist pulses.

In this paper, we propose a more power-efficient signaling scheme by means of adding a time-varying bias to the transmitted PAM signal. The introduced bias is strictly bandlimited and it consists of a sinusoidal and a DC signal with optimized coefficients. The sinusoidal component of the bias can be used at the receiver to improve the symbol time recovery. By this scheme, up to $0.91 \mathrm{~dB}$ gains can be achieved in signal-to-noise ratio (SNR) compared to the previously best known signaling scheme at the same spectral efficiency, which is based on S2.

\section{System Model}

In the absence of optical amplification, the dominating noise sources are thermal noise and shot noise in the photodetector $^{6}$. The IM/DD link can in this case be accurately modeled as a baseband additive white Gaussian noise (AWGN) channel with a nonnegative input signal ${ }^{1}$. Fig. 1 shows the passband model of a IM/DD system (top) and the corresponding baseband channel (middle). We construct the nonnegative transmitted intensity as a modified PAM signal

$$
x^{+}(t)=f(t)+\sum_{k=-\infty}^{\infty} a_{k} p(t-k T),
$$

where $a_{k} \in\{0,1\}$ is the $k$ th transmitted bit, $T$ is the bit time, and $p(t)$ is an arbitrary pulse with bandwidth $B \leq 1 / T$. ISI-free transmission is achieved by considering only Nyquist or root-Nyquist pulses $p(t)$, for sampling or matched-filter (MF) receiver designs, respectively ${ }^{7}$. The nonnegativity constraint is satisfied by adding a proper signal bias $f(t)$ to the PAM signal.

The average transmitted optical power can be computed as ${ }^{1,4}$

$$
P_{\mathrm{opt}}=\frac{1}{T} \int_{0}^{T} \mathbb{E}\left\{x^{+}(t)\right\} \mathrm{d} t,
$$

where $\mathbb{E}\{\cdot\}$ denotes the statistical expectation. In order to ensure eye and skin safeties, and to optimize the energy consumption, the average optical power has to be kept within certain limitations.

\section{The Bias Signal}

The bias signal $f(t)$ can be any waveform, as long as it is strictly bandlimited and achieves the nonnegativity of the transmitted signal. We consider

$$
f(t)=\mu_{0}+\mu_{1} \cos \left(\frac{2 \pi t}{T}+\phi\right),
$$

which is in the form of the Fourier series of any waveform of bandwidth $1 / T$ and period $T$. The coefficients $\mu_{0}, \mu_{1}$ and the phase $\phi$ are constant. For any given $\mu_{1}$, the DC component $\mu_{0}$ can be chosen to ensure $x^{+}(t) \geq 0$. From the average optical power perspective, the cosine component of the bias does not require any extra optical power since its integral in (2) is equal to zero. The extra power is consumed only by the DC bias. However, compared to using only a DC bias ${ }^{5}$, the 


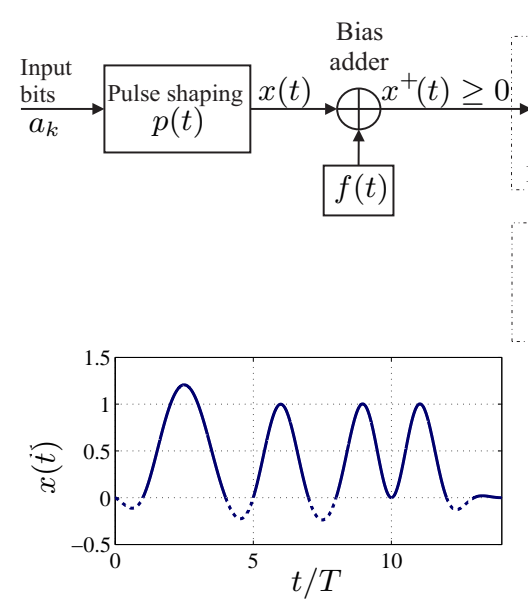

(a)

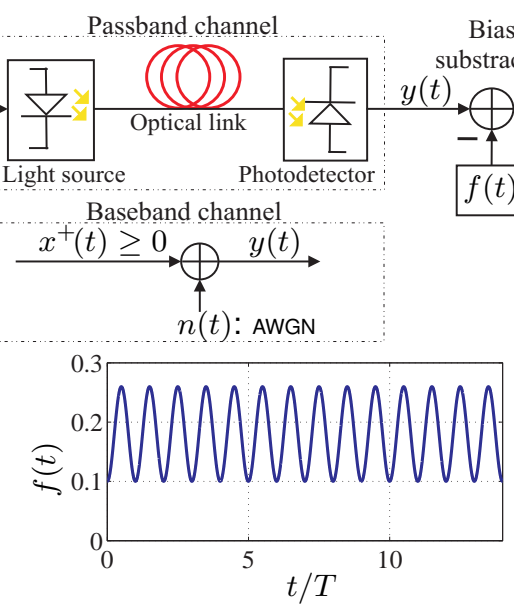

(b)

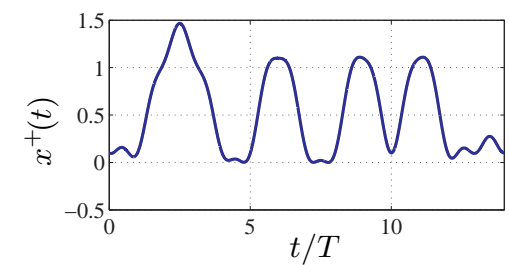

(c)

Fig. 1: The passband IM/DD system model (top). The baseband IM/DD channel (middle). The negative PAM waveform before adding the bias (bottom (a)), where the dashed part represents the negative values; the added bias (bottom (b)); the resultant nonnegative waveform (bottom (c)).

transmission is more power-efficient because less DC bias is required after adding a suitably scaled cosine term. If $\mu_{1} \neq 0$, the bandwidth of $x^{+}(t)$ is $\max (B, 1 / T)=1 / T$, where $B \leq 1 / T$ is the bandwidth of $p(t)$. The receiver does not need any extra synchronization for the time-varying bias, since the cosine term has the same period as the bit clock. Moreover, the cosine-tone added to the spectrum can be used at the receiver in the clock-recovery circuit, analogously to detecting the return-to-zero modulation format which has a similar spectral component at $1 / T{ }^{6}$

Fig. 2 shows the normalized spectrum of a signal $x^{+}(t)$ formed using the proposed modulation format and the root-raised-cosine pulse with a roll-off factor $\alpha=1$ (see Results Sec.). It can be noticed that the spectrum is strictly bandlimited and exhibits a spectral component at $f=1 / T$ which corresponds to the cosine term in (3). A narrow-bandpass filter or a phaselocked loop can easily isolate this component for further use in the clock-recovery circuit.

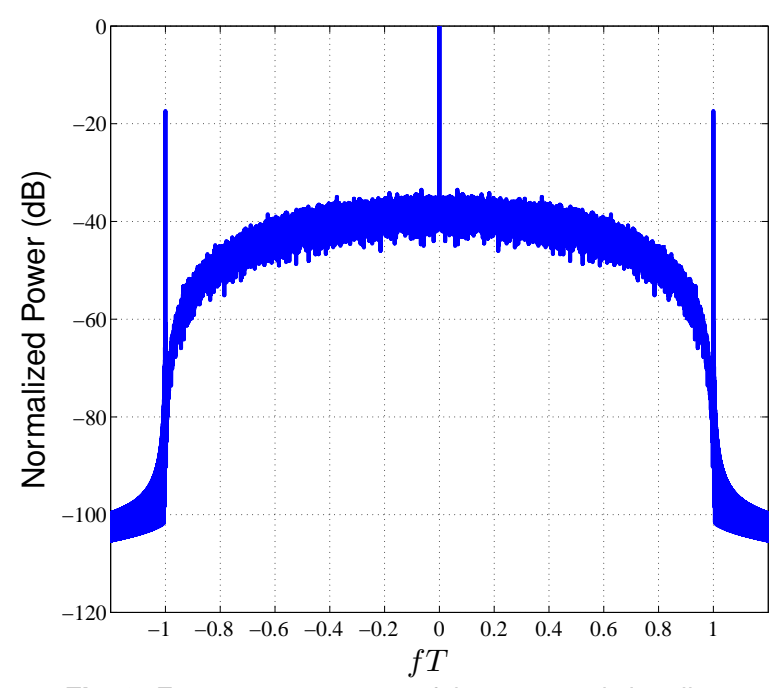

Fig. 2: Frequency spectrum of the proposed signaling method using a root-raised-cosine pulse with $\alpha=1$.
By finding an expression for the global minimum of $x^{+}(t)$ that is independent of the instantaneous transmitted data bits $a_{k}$, an optimization similar to Tavan ${ }^{5}$ is formed and numerically solved to obtain the $\mu_{0}, \mu_{1}$, and $\phi$ that maximize the optical power efficiency.

\section{Results}

Tab. 1 presents the achievable optical gains obtained by using the proposed signaling method for different pulse shapes $p(t)$ in terms of asymptotic power efficiency (APE) defined as

$$
\mathrm{APE}=\frac{P_{\mathrm{opt}}^{\mathrm{ref}}}{P_{\mathrm{opt}}},
$$

where $P_{\mathrm{opt}}^{\mathrm{ref}}$ is the average optical power of the benchmark signaling method ${ }^{3}$, employing a sampling receiver and the S2 pulse, which does not require any bias signal. The S2 pulse is the most power-efficient pulse previously known for the bandlimited IM/DD channel $^{3}$ and requires the same bandwidth as our proposed signaling scheme, i.e., $B=1 / T$, which leads to a fair comparison. Results are given for the raisedcosine $(R C)^{7}$, the first-order parametric linear $(P L)^{8}$, the better than Nyquist $(\mathrm{BTN})^{9}$, and the first-order $\mathrm{Xia}^{10}$ pulses. When the MF receiver is used, the root-Nyquist pulses are obtained from the Nyquist pulses by taking the square root in the frequency domain ${ }^{7}$. The excess bandwidth for each pulse is controlled by the roll-off factor parameter $\alpha \in[0,1]$. The optimal APE for different pulses is obtained at $\alpha=1$, except for PL ( $\alpha=0.992$ ) and BTN $(\alpha=0.976)$ in case of using the MF receiver. Except the Xia pulse, which is the only nonsymmetrical pulse in the time domain, the optimal phase of the cosine term is $\phi=0$.

The results in Tab. 1 show that using the proposed bias signal, the MF receiver is more power-efficient than the sampling receiver, in contrast to previous results $^{3-5}$. The best APE is obtained by either the RC or Xia pulse using a MF receiver. They have the same 
Tab. 1: The achievable APE (dB) of the proposed method for various pulses and their corresponding bias coefficients. The highlighted cells have the best APE.

\begin{tabular}{|c|c|c|c|c|c|}
\hline & $f(t)$ & $\mathrm{RC}$ & $\mathrm{PL}$ & BTN & Xia \\
\hline \multirow[t]{2}{*}{$\begin{array}{l}\text { Sampling } \\
\text { receiver }\end{array}$} & $\mu_{0}$ & $\begin{aligned} \mathrm{APE} & =-0.277 \\
\mu_{0} & =0.033\end{aligned}$ & $\begin{array}{c}\mathrm{APE}=0 \\
\mu_{0}=0\end{array}$ & $\begin{aligned} \mathrm{APE} & =-0.039 \\
\mu_{0} & =0.005\end{aligned}$ & $\begin{aligned} \mathrm{APE} & =-1.893 \\
\mu_{0} & =0.273\end{aligned}$ \\
\hline & $\mu_{0}+\mu_{1} \cos \left(\frac{2 \pi t}{T}+\phi\right)$ & $\begin{aligned} \mathrm{APE} & =-0.257 \\
\mu_{0} & =0.031 \\
\mu_{1} & =0.011\end{aligned}$ & $\begin{array}{c}\mathrm{APE}=0 \\
\mu_{0}=0 \\
\mu_{1}=0\end{array}$ & $\begin{aligned} \mathrm{APE} & =-0.032 \\
\mu_{0} & =0.004 \\
\mu_{1} & =0.004\end{aligned}$ & $\begin{aligned} \mathrm{APE} & =-1.051 \\
\mu_{0} & =0.137 \\
\mu_{1} & =0.136\end{aligned}$ \\
\hline \multirow[t]{2}{*}{$\begin{array}{l}\text { Matched } \\
\text { filter } \\
\text { receiver }\end{array}$} & $\mu_{0}$ & $\begin{aligned} \mathrm{APE} & =-0.388 \\
\mu_{0} & =0.273\end{aligned}$ & $\begin{aligned} \mathrm{APE} & =-0.771 \\
\mu_{0} & =0.345\end{aligned}$ & $\begin{aligned} \mathrm{APE} & =-0.905 \\
\mu_{0} & =0.371\end{aligned}$ & $\begin{aligned} \mathrm{APE} & =-0.388 \\
\mu_{0} & =0.273\end{aligned}$ \\
\hline & $\mu_{0}+\mu_{1} \cos \left(\frac{2 \pi t}{T}+\phi\right)$ & $\begin{array}{c}\mathrm{APE}=0.454 \\
\mu_{0}=0.137 \\
\mu_{1}=0.136\end{array}$ & $\begin{array}{c}\mathrm{APE}=0.171 \\
\mu_{0}=0.180 \\
\mu_{1}=0.166\end{array}$ & $\begin{array}{c}\mathrm{APE}=0.010 \\
\mu_{0}=0.205 \\
\mu_{1}=0.169\end{array}$ & $\begin{array}{c}\mathrm{APE}=0.454 \\
\mu_{0}=0.137 \\
\mu_{1}=0.136\end{array}$ \\
\hline
\end{tabular}

optimal bias because at $\alpha=1$, the Xia pulse is a time-shifted version of the root-RC pulse by $-T / 4$. Hence, with a $\phi=-\pi / 2$-radian shift in the cosine term, the same APE can be achieved for both of the pulses.

Fig. 3 illustrates the bit error rate (BER) of the system for the $\mathrm{S} 2$ and $\mathrm{RC}$ pulses as a function of the required SNR defined as ${ }^{1} \mathrm{SNR}=P_{\mathrm{opt}}^{2} /\left(N_{0} R_{b}\right)$, where $R_{b}=$ $1 / T$ is the bit rate and $N_{0} R_{b}=N_{0} B$ the in-band noise power. A gain of $0.91 \mathrm{~dB}$ can be noticed, which is twice the APE gain, between our proposed signaling method compared to the previously best known scheme.

\section{Conclusion}

This paper presents a power-efficient signaling method for bandlimited optical intensity transmission. A timevarying but bandlimited bias signal, consisting of a DC and a cosine term, is added to the transmitted signal to make it nonnegative. The proposed method can simplify the symbol time recovery at the receiver and enables power-efficient use of root-Nyquist pulses and the matched-filter receiver design. The evaluation of the proposed modulation format is done by computing the asymptotic power efficiency and the BER of the system. The results show that gains up to $0.91 \mathrm{~dB}$ can

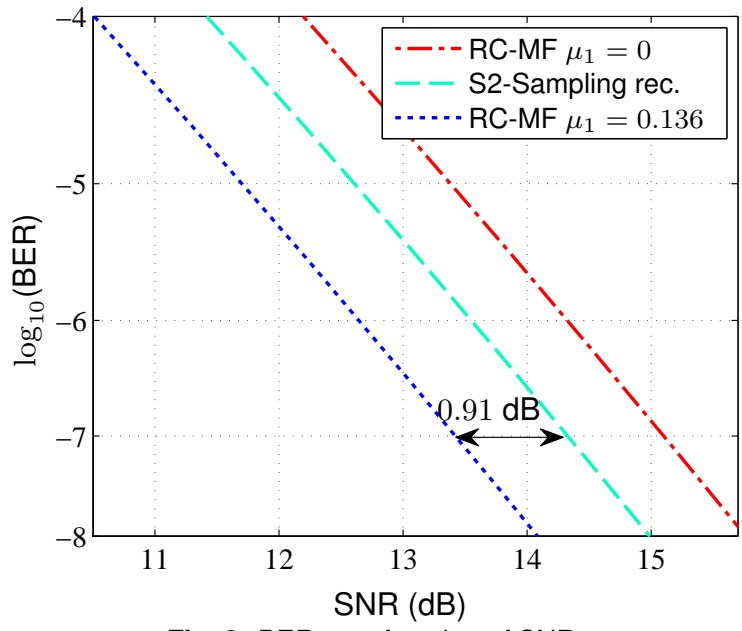

Fig. 3: BER as a function of SNR. be achieved in SNR compared to the best previously known signaling method at the same bandwidth.

\section{References}

[1] J. M. Kahn and J. R. Barry, "Wireless Infrared Communications," Proc. IEEE, Vol. 85, no. 2, p. 265 (1997).

[2] S. Randel et al., "High-Speed Transmission over Multimode Optical Fibers," OFC, paper OWR2 (2008).

[3] S. Hranilovic, "Minimum Bandwidth Nyquist and Root-Nyquist Pulses for Optical Intensity Channels," IEEE GlobeCom, Vol. 3, p. 1368 (2005).

[4] S. Hranilovic, "Minimum-Bandwidth Optical Intensity Nyquist Pulses," IEEE Trans. Commun., Vol. 55, no. 3, p. 574 (2007).

[5] M. Tavan et al., "Bandlimited Intensity Modulation," IEEE Trans. Commun., Vol. 60, no. 11, p. 3429 (2012).

[6] G. P. Agrawal, Fiber-Optic Communication Systems, 4th ed., John Wiley \& Sons (2010), p. 148157.

[7] J. G. Proakis and M. Salehi, Digital Communications, 5th ed., McGraw-Hill (2008), ch. 9.

[8] N. C. Beaulieu and M. O. Damen, "Parametric Construction of Nyquist-I Pulses," IEEE Trans. Commun., Vol. 52, no. 12, p. 2134 (2004).

[9] N. C. Beaulieu et al., "A 'Better Than' Nyquist Pulse," IEEE Commun. Lett., Vol. 5, no. 9, p. 367 (2001).

[10] X. G. Xia, "A Family of Pulse-Shaping Filters with ISI-Free Matched and Unmatched Filter Properties," IEEE Trans. Commun., Vol. 45, no. 10, p. 1157 (1997). 